\title{
herm3TIC-tv: Prototype of a human and social sciences laboratory based on Deleuze-Guattari philosophy and the application of the new ICT
}

\section{SIGRADI2018 TECHNOPOLITICAS \\ xxii congresso da sociedade iberoamericana de gráfica digital 22th conference of the iberoamerican society of digital graphics $07|08| 09 \mid$ novembro|2018 iau usp | são carlos | sp br}

\author{
Manuel Cebral Loureda \\ Santiago de Compostela University | Spain | man.cebral@gmail.com
}

\begin{abstract}
Our social interactions, increasingly mediated by technology, require subjectivity singularization spaces. The philosophy of Deleuze-Guattari gives us the keys of a machinic device that can counter-actualize the generic arrengement we suffer, derived from media and technology. This space is considered as a scenic space but also scientific and analytical, developing what Deleuze-Guattari called schizoanalysis clinic but that we can also understand as the development of a proper and interdisciplinary methodology for the new laboratories of human and social sciences.
\end{abstract}

Keywords: Schizoanalysis; Visual arts; Interactives; Big data; Neuroimaging.

\section{INTRODUCCIÓN}

El proyecto define la posibilidad de un laboratorio para ciencias humanas y sociales como demanda compartida desde los primeros laboratorios de psicología, pasando por las prácticas de colectivos de artistas como el surrealista, hasta las más recientes aplicaciones de las ciencias humanas y sociales para el estudio de hábitos y costumbres de la población mediante grandes volúmenes de datos. Desde entonces hasta ahora, las ciencias humanas expresan una necesidad común de darle forma a prácticas que les permitan ir más allá de la documentación hacia la experimentación. La posibilidad de este laboratorio se concreta como desarrollo de la propuesta de Deleuze-Guattari $(2002 ; 2011 ; 2017)$ de una clínica de esquizoanálisis que es llevada a la práctica en un trabajo interdisciplinar entre ingeniería de la comunicación, artes escénicas y visuales, filosofía y psicología.

Si bien la filosofía contemporánea ha deconstruido los conceptos de Verdad, Historia y Sujeto con mayúsculas, como grandes relatos (Lyotard, 1999; Vattimo, 1990), Foucault y Deleuze-Guattari redefinen a su vez en el postestructuralismo el archivo y los estratos históricos dándole una nueva oportunidad a la historia ahora escrita con minúsculas. Partiendo del análisis del saber como arqueología, Foucault (2005) relativiza el concepto de verdad como algo producido en cada época histórica, del mismo modo que la idea de hombre. Historia y hombre son producidos en el seno de unas relaciones de saber y de poder que, mediante una serie de dispositivos enunciativos y de visibilidad, construyen las legitimidades propias de cada época.

Deleuze-Guattari recogen este análisis postestructural y redefinen los estratos históricos como superficies libidinales de lo que denominan cuerpo sin órganos (2017; 2002). Si bien los archivos foucaultianos tenían la capacidad de recrear los aspectos coercitivos de cada época, los dispositivos que nos obligaban a ver y decir de un determinado modo, Deleuze-Guattari intentan ir más allá, hacia los elementos productivos y creativos que los conforman. Que haya unas condiciones que en cada época producen la verdad y al hombre (históricamente en masculino) de la misma, no nos limita tanto a entenderlos como coerciones absolutas sino que nos permite entender sus alternativas; lo que Deleuze-Guattari denominan líneas de fuga. De este modo, las líneas de fuga se convierten en vectores de desterritorialización, es decir, fuerzas creativas que marcan los índices de libertad y novedad para cada época o estrato histórico. Para comprender las épocas históricas y sus composiciones, debemos de observar tanto las fuerzas que nos coaccionan y territorializan formando estos estratos, como aquellas que nos desterritorializan y nos permiten crear nuevos espacios; en un principio lisos, nómadas y cargados de ricas potencialidades. Estos espacios pueden posteriormente ser de nuevo territorializados, estratificados, una vez que se haya asimilado su novedad, en un movimiento sucesivo de regeneración, creación y excepcionalidad. 


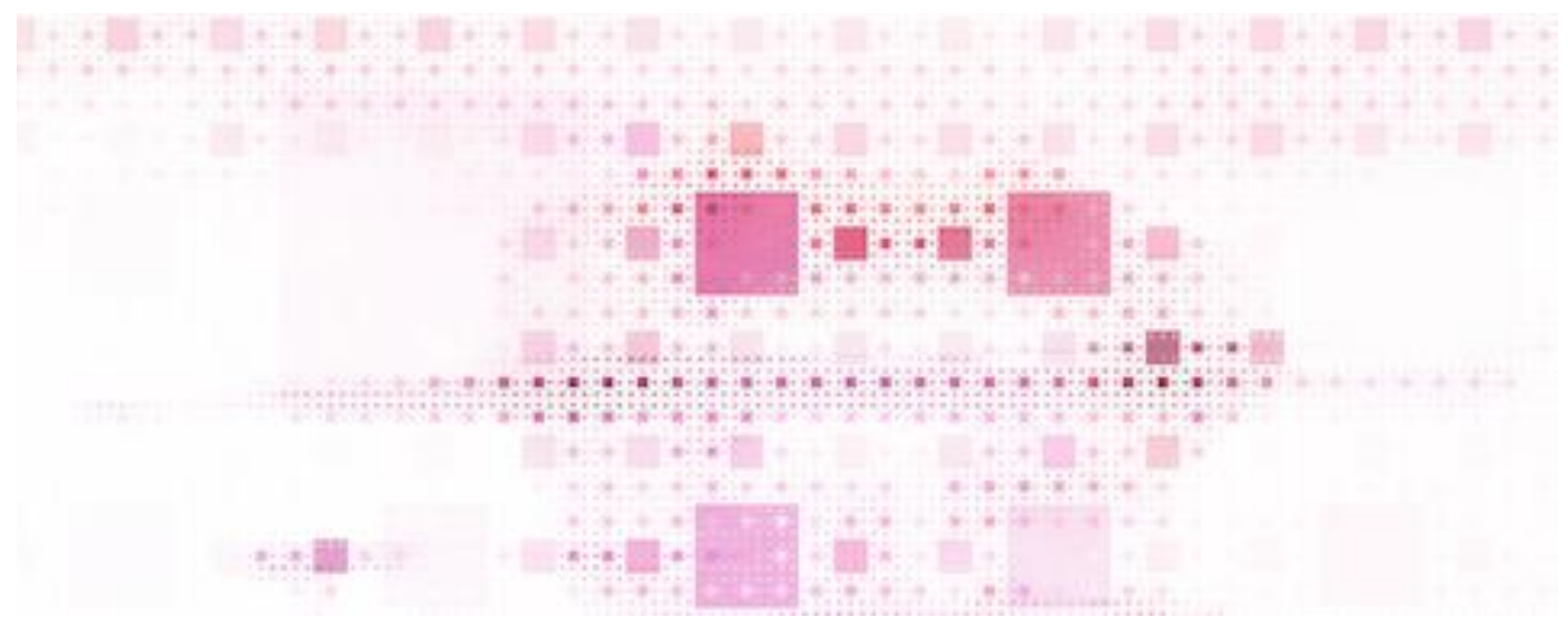

Figura 1: digitalización de interfaz transformando, según el contraste, píxeles en activadores durante una sesión de herm3TICa-tv. Fuente: Autor.

De este modo, Deleuze-Guattari, siguiendo la estela de Foucault, se alejan por tanto del postmodernismo, dando cierta validez al estudio de las épocas históricas y sus archivos. El propio Deleuze (2003) llama a Foucault un nuevo archivista ya que en su metodología, el estudio de archivos históricos y sociales son la base sobre la cual se asientan sus análisis y argumentos. La idea es que no existe otra cosa que esos archivos y sus reacciones ante ellos; es decir, no hay una idea, espíritu de la época o intención que se exprese por detrás de ellos; sino más bien el modo en que esos archivos de hecho nos condicionan. También los estudios de Deleuze-Guattari se sirven de la perspectiva histórica, así claramente en Antiedipo, (2017) donde se desarrolla una genealogía de las máquinas sociales como en Mil Mesetas (2002), donde la historia es citada no como una línea en desarrollo, sino como instantes concretos o mesetas que nos hablan de ciertos momentos fulgurantes y relativamente fugaces en los que se produce una especie de novedad en cierta dimensión de la línea histórica del tiempo. Cada capítulo o meseta en este texto, es fechado aludiendo a un acontecimiento destacable que se sale fuera del orden cronológico de la Historia (con mayúsculas) hacia una atemporalidad (o intempestividad propia del pensamiento en tanto que tal) que Deleuze (2011) ya había bautizado como Aión, recuperando el término griego.

Sin embargo, el acercamiento tanto de Foucault, como de Deleuze-Guattari a la historia, no es suficiente para realmente poder ubicar sus filosofías en la otra gran corriente de la filosofía contemporánea: la Hermenéutica. Surgida como desarrollo a la filosofía existencial heideggeriana, Gadamer, principalmente, sienta las bases de la hermenéutica como filosofía contemporánea que se caracterizará por acercar el análisis de la facticidad heideggeriana al contexto de la interpretación históricamente situada, dando lugar a una nueva noción de verdad que se produciría en esa nueva fusión de horizontes (Gadamer, 2002). Es decir, simplificando un poco, podemos decir que la verdad es una consecuencia interpretativa de una re-contextualización histórica que, por otro lado, es constante, abierta e infinita (jamás se termina, nunca se alcanza una última interpretación). Esta noción de verdad y esta concepción de la historia, tiene ciertos rasgos en común con la facticidad postestructural de los archivos planteada tanto por Foucault como por
Deleuze-Guattari; pero obviamente, se alejan bastante, al mismo tiempo, a la hora de concebir el lugar en el que esto acontece. En el caso de Foucault, no se especifica demasiado cuál sería ese lugar y el modo en que sería posible una comprensión y reinterpretación (Baudrillard, 2001). Sí que queda más claro, sin embargo, en el caso de Deleuze y Guattari, que proponen la pragmática del esquizoanálisis, un espacio en el que la verdad en vez de ser reinterpretada y comprendida por un sujeto, es producida en un dispositivo maquínico. Para marcar esta diferencia, aunque con cierto guiño también a la hermenéutica, escogemos el neologismo herm3TICa para significar este proyecto.

La herm3TICa sería un acercamiento a la interpretación histórica pero fuera del marco de la verdad y el sujeto propuestos por la hermenéutica filosófica. Como hemos dicho, la noción de verdad, en vez de ser interpretada, pasa a ser producida; y en vez de darse en un sujeto o una conciencia pasa a darse en (o a través de) un dispositivo o máquina. De ahí el uso del acrónimo TIC. Pero también es un gesto hacia la otra gran tradición que comparte esta etimología: la hermética de donde incluso autores muy próximos a la hermenéutica como Ortíz-Osés sugirieron un acercamiento. En concreto a través del concepto hermético-hermenéutica Ortíz-Osés (2003) se refiere a la necesidad de quedarse en un punto intermedio de las dos tradiciones porque una sola de ellas en exclusiva siempre sería parcial y limitada. Pero también una de las grandes influencias deleuzianas en el estudio de la tecnología, Simondon (2017), defiende la hermética como la tradición que primero hace un uso cognitivo de la tecnología. Hay otros autores que también han desarrollado lecturas herméticas de la filosofía de Gilles Deleuze (Ramey, 2016) aunque en este caso no desde la perspectiva tecnológica que nos ocupa en este proyecto.

\section{METODOLOGÍA}

El proyecto herm3TICa-tv ha sido realizado como prototipo de un laboratorio para ciencias humanas y sociales o laboratorio de esquizoanálisis en la denominación de Deleuze-Guattari (2017) en el que el paciente (lo pondremos siempre en cursiva para recordar que no es un sujeto pasivo como podría entenderse) interactúa en una 


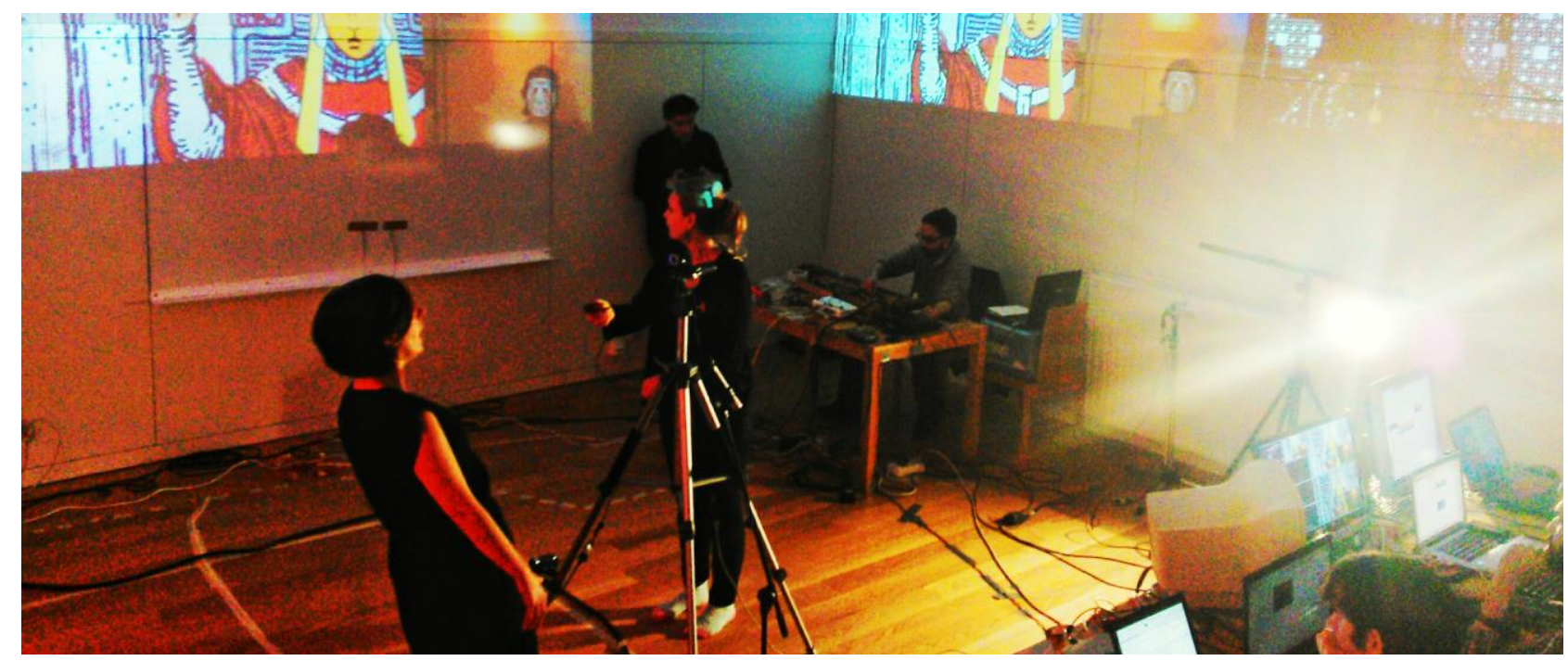

Figura 2: plató digital de producción durante una sesión en el laboratorio herm3TICa-tv. Fuente: Autor.

especie de cabina o plató digital de creación interactivo. Utilizando el audiovisual de manera creativa y en conexión streaming generamos un interfaz de enunciación que trasciende el sujeto y permite la aparición de subjetividades en su relación con archivos históricos y/o sociales. Sobre esta base, el proyecto pretende ahora incorporar las capas de Big data y el análisis de neuroimagen para una verdadera interacción sensible e informacional de los usuari@s o pacientes con su estructura o máquina social, con la posibilidad de trabajar también sobre archivos (textos, imágenes, etc.) de otras épocas; pero siempre desde nuestro presente.

\section{CAJA DE RESONANCIA}

El plató digital está diseñado a modo de caja de resonancia, físicamente cerrada y por eso también hermética. Sin embargo, al mismo tiempo que cerrada, la tradición hermética proclama lo abierto e interactivo, pues el conocimiento de sus principios permite, supuestamente, la interconexión de planos y realidades, básicamente macrocosmos y microcosmos. Sin justificar aquí, o no, esta tradición, el proyecto sí que asume que las tecnologías nos abren los espacios físicos a nuevas dimensiones, siendo de hecho esa una de las concepciones más fidedignas de lo virtual (Levy, 1999). A través de la información y el audiovisual las tecnologías nos interconectan y nos permiten interactuar en dimensiones no físicas, pero quasifísicas, relativizando lo abierto y lo cerrado, lo aislado y lo conectado, lo micro y lo macro. Las resonancias de todas las dimensiones, sus interacciones comunes, son en sí mismas, la actividad del laboratorio, es su producto al mismo tiempo que su objeto, acercándonos al concepto también deleuze-guattariano de máquina sintetizadora que más allá juicio sintético kantiano, ubicado todavía en el Sujeto y el Yo, se reubica en la impersonalidad de los agenciamientos maquínicos histórico-sociales. Algunos autores recientes (Bratton, 2015) aplican de hecho diagramas de deleuze-guattarianos semejantes al que aquí planteamos para explicar interacciones entre capas de computación a nivel planetario. En nuestro caso, el acento está puesto en la capacidad de contra-efectuación y singularización del usuari@, como veremos.

A nivel propiamente metodológico, podemos ordenar estas resonancias o interacciones del plató digital o cabina de esquizoanálisis en torno a 4 espacios a su vez interrelacionados e interdependientes. Se inspiran en los 4 espacios escénicos según la división que propone Marta Schinca (2011a; 2011b), reelaborando nociones previas de Rudolph von Laban, Rudolph Bode, y Jean Jacques Dalcroze en ámbitos de teatro contemporáneo y teoría del movimiento; y que se utilizan a su vez en herm3TICa-tv como base sobre la que desarrollar el laboratorio.

\section{ESPACIO DE INTERACCIÓN 1. INTERACCIÓN CON EL PROPIO CUERPO: EL CUERPO SIN ÓRGANOS Y LOS BIODATOS}

Es el modo como sentimos nuestro propio cuerpo. Lo hacemos corresponder en herm3TICa-tv con outputs que produce el propio cuerpo, nuestros correlatos inmediatos, o lo que Deleuze-Guattari denominaron cuerpo sin órganos. Sin que emitamos ninguna acción, el cuerpo es de por sí productivo, reposa sobre sus intensidades vitales tales como el ritmo cardíaco, la temperatura, presión arterial, humedad, etc. que podemos medir con diferentes sensores. Entre otras cosas, lo que el esquizoanálisis reprocha al psicoanálisis es la idea de que en el fondo de nuestra vitalidad resida algo como un instinto de muerte (Deleuze-Guattari, 2017, p. 343). Por el contrario, el grado cero de nuestro cuerpo es su simple vitalidad, que es ya algo: es el plano sobre el que todo se constituye, donde todo aparece y es posible, en un espacio diferencial e intensivo que es el del cuerpo sin órganos. Vértigos, escalofríos, devenires, aceleraciones... son los movimientos del cuerpo sin órganos. En el laboratorio, sus mediciones, además de ser directamente visualizadas en infografías, pueden ser exportadas como nuevas fuentes puestas en interacción con otras mediciones, pues como veremos, todos los espacios interactúan.

\section{ESPACIO DE INTERACCIÓN 2. EL MOVIMIENTO DE NUESTRO CUERPO: LA IMAGEN-ACCIÓN}

Es nuestra capacidad de realizar acciones. Aquello que Deleuze (1994) en sus estudios sobre cine, denominó imagen-acción: saltar, desplazarnos, agacharnos, arrastrarnos, caernos, correr, andar... son acciones que nos permiten movernos de manera más o menos fácil en nuestro laboratorio. Estando la caja de resonancia videovigilada con cámaras de captura, creamos mediante software espacios de esas capturas que se activan o 
desactivan cuando pasamos por ellos. O bien podemos crear interacciones no por zonas de encuadre, sino por cantidad de movimiento. En algunos de los prototipos realizados, mover un brazo, por ejemplo, rápidamente, podía ralentizar o acelerar un clip que se estaba utilizando. Además de las cámaras de captura, la caja de resonancia cuenta con monitores que devuelven al usuari@ que interactúa o paciente diferentes perspectivas e interfaces de información de lo que está pasando. La propia captura de lo que hace y cómo se mueve, pero también, según las escenas en las que estemos trabajando, otras fuentes de audiovisual o visualizaciones de información en cada caso, por lo que las acciones dentro del laboratorio no se limitan a su supuesto efecto físico sensoriomotor. Por el contrario, nuestros movimientos y acciones repercuten a través de los dispositivos programados en activaciones 0 modulaciones en otros niveles; lo cual nos lleva a la implicación del siguiente espacio de interacción.

\section{ESPACIO DE INTERACCIÓN 3. EL ESPACIO ESCÉNICO TOTAL: LA PROPIOCEPCIÓN}

La cámara en su totalidad compone un plano de enunciación que emitimos por streaming. En ese plano pueden converger diferentes fuentes: encuadres de lo que acontece en la cabina de interacción, fuentes externas de otros clips que estemos utilizando o incluso visualizaciones de datos e información. Este plano conjunto a la vez que es emitido es devuelto al interior de la cabina de modo que el paciente puede percibir cómo afectan sus movimientos en los diferentes espacios y con los diferentes dispositivos a la composición final de la emisión. Es un plano de composición de la imagen, un plano sensible, que se mide por su expresividad estética y su carácter principalmente audiovisual, aunque suele incluir textos. De este modo, la acción como tal deja de tener importancia por su efectividad motora para ser reinterpretada por la propiocepción o el feedback que produce compositivamente en la interacción con otros planos. Del mismo modo que Deleuze propone una lectura de la imagen-acción en la imagen-tiempo (1984), el producto compositivo del laboratorio que se emite en streaming más que narrar ningún acto nos muestra una intensidad de relaciones, nos obliga a vernos desde fuera al mismo tiempo que hacemos algo, es decir, pensar la totalidad, el conjunto al mismo tiempo que la acción con la que concretamente manipulamos algo. Esta simultaneidad de lo concreto y lo total en la percepción, tiene que ver con esa cualidad propioceptiva que muchos psicólogos han apuntado como la clave que nos obliga a aprender con todo el cerebro (Williams, 1986); y también es afín, como veremos, a la concepción topológica del cerebro que terminan proponiendo Deleuze-Guatttari (2011).

\section{ESPACIO DE INTERACCIÓN 4. EL ESPACIO IMAGINARIO DE VISUALIZACIÓN}

Además de las fuentes capturadas con cámaras sobre el movimiento y las interacciones con sensores (así como sus respectivas digitalizaciones, codificaciones y recreaciones audiovisuales posibles) incluimos también fuentes externas que tienen que ver con nuevas opciones de visualización que podemos proyectar en escena. Es ya un recurso escénico habitual y extendido entre actores, que visualizen interiormente ciertas imágenes; se utilizan a modo de inspiración para trasladarse psicológicamente en escena y adquirir así una tonalidad o intensidad actoral precisa. Si bien esto es una técnica actoral, define propiamente un espacio escénico en modelos como el de Marta Schinca, dándole la importancia de un nuevo espacio más que conviene habitar para una adecuada cualidad escénica. Ahora bien, desde que el audiovisual entra en escena, cada vez más es utilizado como recurso que nos traslada en un sentido muy semejante a nuevos espacios, texturas y sensaciones a través de visualizaciones que de facto ya se pueden reproducir audiovisualmente. Son normalmente y cada vez más opsignos y sonsignos (Deleuze, 1984), es decir, más que espacios muy reconocibles, o representables cualidades ópticas y sonoras de los mismos, que nos permiten habitar de un modo más sensible e inmersivo estos espacios en vez de simplemente evocarlos desde la distancia. El espacio imaginario y de visualización explicita por tanto estas fuentes, poniendo en relación lo que ocurre físicamente en la caja de resonancia y lo imaginario, permitiendo que ambos planos sean de algún modo intercambiables.

Hasta aquí los espacios escénicos de interacción tal como se redefinen en los prototipos realizados en herm3TICa-tv. Sobre ellos se pretenden ahora implementar dos nuevos espacios de interacción:

\section{ESPACIO DE INTERACCIÓN 5. INTERACCIONES SOCIALES EXTERNAS Y BIG DATA}

Como toda emisión en streaming y en red, el laboratorio genera un impacto público a través de internet que se puede difundir y ser utilizado en todo tipo de redes sociales. De hecho, el propio laboratorio tiene la posibilidad de funcionar como red social. Al mismo tiempo que se emiten las sesiones de esquizoanálisis, usuari@s extern@s pueden comentar y valorar las emisiones generando múltiples posibilidades de feedbacks generadores de nuevo contenido. Ese contenido, podríamos dividirlo en tres grandes tipos de interacción:

- $\quad$ Simples valoraciones de las emisiones: al modo de me gusta, valoraciones de estrellas u otras.

- Valoraciones con opciones de comentarios: en los que se pueden incluir hastags que ayuden posteriormente a identificar, ordenar y visualizar tendencias.

- Interacciones con la propia cabina de esquizoanálisis: de modo que los usuari@s (probablemente ciertos usuari@s con una vinculación especial con el proyecto) dispongan de medios que les permitan variar las condiciones interiores de la cabina y/o interaccionar directamente con el paciente. De hecho, es posible que este tipo de interacciones requieran hardware especializado para el usuario, de modo que pueda transmitir elementos de su propia fisicidad a través de sensores o accionar mecanismos mediante mandos. En experiencias que nos pueden recordar a las planteadas ya al comienzo de la web por Lanier (2011) o Lévy (1997).

Obviamente todas estas interacciones, sobre todo las de los dos primeros tipos, generan a su vez nuevos planos infográficos que, en este caso, pueden ser reutilizados como nuevas fuentes compositivas que serían devueltas tanto interiormente a la caja de resonancia como fuentes publicables en redes o en el propio streaming que 

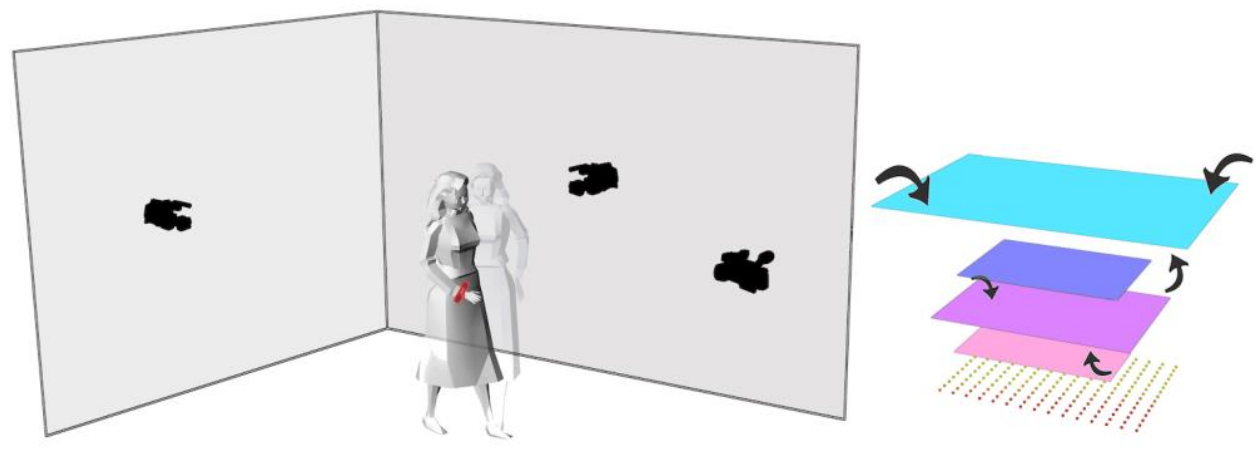

Interacción con datos de redes sociales y Big data

interfaz de emisión streaming plano de enunciación

Espacio de interacción 4: superposición de fuentes imaginario Espacio de interacción 3: propiocepción y composición de escena Espacio de interacción 2 imagen-acción

Espacio molecular de interacción 1 : sensores y biodata

Figura 3: diagrama del laboratorio videovigilado junto a las relaciones de capas de interacción. Fuente: Autor.

generamos. De este modo, podemos tener un feedback en tiempo real de lo que hacemos y sobre todo, poder intervenir sobre esa información y/o su visualización o materialización como input en la caja de resonancia. Como veremos en el apartado de tratamiento de resultados, la información genérica corresponde más a la tipología social y la opinión frente a la cual, el motivo del proyecto es dar la posibilidad de contra-efectuación y singularización de los pacientes. El Big data y las fuentes que podamos generar a modo de inputs en la caja de resonancia funcionan por tanto como una capa más sobre la que intervenimos, una nueva materialidad que podemos tocar y modular al ser transformada en una fuente sensible (un sonido, una tonalidad en la imagen, un nivel en un gráfico, etc.).

\section{ESPACIO DE INTERACCIÓN O. LA NEUROIMAGEN}

Si bien comenzamos definiendo el espacio de interacción más inmediato aquel en el que el cuerpo interacciona consigo mismo, y de este modo, variables biológicas podían generar de por sí un grado cero de latencia, involuntario, pero siempre con alguna positividad intensiva; verdaderamente podemos ir un poco más allá (o más acá) a algo que está previamente: el propio sistema nervjoso y el cerebro (van den Heuvel, Sporns, 2013). Las técnicas de neuroimagen aplicadas sobre el paciente constituyen un cero absoluto aún por investigar en el contexto de herm3TICa-tv pero nos pueden dar la posibilidad de llegar un poco más profundo en el análisis psicológico y corroborar el hecho de que realmente nuestra cabina funciona como laboratorio analítico. ¿Cómo reacciona el sistema nervioso y el cerebro en un espacio escénico y aumentado, generador de sentido, como el que planteamos? ¿Cómo reacciona el cerebro en un espacio videovigilado y propioceptivo a nivel visual, pero también a nivel sensorial e incluso social? En este sentido, ¿cómo cambia nuestro pensamiento al vernos retratados en redes sociales? ¿Cómo se generaliza y se tipifica nuestra opinión y cómo podemos contrarrestar estos movimientos? Todas estas preguntas sólo tienen sentido si el desarrollo del laboratorio es capaz no sólo de evitar la sobre-exposición de sus pacientes en los medios, sino de imaginar y confabular con ellos. El destino de todo esquizoanálisis no es la homogeneización, el tipo genérico hombre, ni siquiera la individualización frente a la masa; sino la singularización radical, los devenires animales, femeninos, minoritarios e imperceptibles (Deleuze-Guattari, 2002), la potencialidad menor de lo que deviene y muta frente a la tipología mayor.
En su texto ¿Qué es la filosofía?, (Deleuze-Guattari, 2011) entienden el cerebro como la junción (que no la unidad) de arte, ciencia y filosofía; y de manera semejante, la neuroimagen posibilita en herm3TICa-tV también una junción de estas perspectivas. Científicamente, la neuroimagen nos acerca analíticamente a lo que sucede en nuestro sistema nervioso; pero sus estímulos son sobre todo originados como reacción a la exposición sensible que es la cabina de resonancia. Por último, sus resultados deben de poder hacernos entender qué nos pasa, cómo somos, cómo reaccionamos y cómo deseamos de manera más consistente; que es lo propio de la filosofía. El cerebro como junción de estas tres realidades, es un cerebro topológico, que más que determinar tipos o formas a priori, es "inobjetivable" (Deleuze-Guattari, 2011, p. 211), permitiendo al contrario hacer aparecer aquellas zonas que se activan anómalamente, nuevas configuraciones a estudiar y en las que profundizar precisamente en tanto que se escapan de lo previamente esperable. Es así que el esquizoanálisis en general y herm3TICa-tv en particular buscan la proliferación de singularidades y sus asociaciones laterales en vez de su tipología clínica arborescente.

\section{NO REVERSIBILIDAD Y SUCESIVIDAD DE LAS SESIONES Y EL PROYECTO}

Por último, un aspecto no señalado todavía del proyecto es el carácter acumulativo y no reversible de las sesiones en el proyecto; e incluso del proyecto mismo. Es decir, hay una cierta tendencia entrópica de herm3TICa-tv como sistema, pero al mismo, se plantea como una manera de atestiguar que esta irreversibilidad nunca es limitante, es decir, que siempre es generadora de nuevas posibilidades e incluso de mayor potencialidad a la hora de poder crear, escoger, componer y singularizar las sesiones y los pacientes en las mismas. Este es un mecanismo que facilita la propia metodología de las cámaras de captura en el espacio videovigilado haciendo que los archivos generados en determinado momento de una sesión puedan ser retrotraídos posteriormente como nuevos planos o capas sobre los que interactuar, convirtiendo de este modo las reacciones contingentes de determinada escena en fuente material y soporte de nuevas escenas. Estos materiales pueden ser:

- Clips con fragmentos de improvisación de movimiento y reacciones a determinados estímulos, la interacción con la tecnología y los dispositivos de la caja de resonancia en general son utilizados como secuencias sobre las que los pacientes se contra-efectúan a sí mismos pasando a 
ser material proyectado en el interior de la cabina y por tanto nuevas fuentes sensibles de contacto, modulación y manipulación.

- De estas nuevas interacciones surgen gestos más concretos que se seleccionan como posiciones clave que articulan completamente escenas posteriores convirtiéndose en espacios de intensidad concreta. Habitar un gesto fortuito, profundizar en nuestras reacciones y tics como espacioes en sí mismos y el modo en que los podemos hacer variar según sus regitros de biodatos que aluden el espacio de interacción 1.

- Estas mediciones nos pueden plantear también configuraciones de referencia tratando de generar finalmente una carta o mapa de los pacientes para cada sesión; pero que nos permitan también asociaciones clínicas laterales que evidencien soluciones o tácticas más que cuadros personalizados.

\section{RESULTADOS}

Básicamente los resultados del proyecto son los propios archivos audiovisuales generados en las sesiones de herm3TICa-tv y que fueron emitidos en streaming en su momento, son posteriormente publicados en la red y categorizados según las escenas que desarrollan y los participantes y pacientes que intervienen. Este material audiovisual por sí mismo, tiene un valor estético, expresivo, ha sido exhibido en alguna exposición en centros de arte y cultura. Además, de estas experiencias hemos recogido testimonios de los participantes bien como pacientes bien como artistas, programadores o cámaras. Los testimonios han sido testimonios siempre testimonios positivos. Algunas cosas que se han destacado han sido:

- Lo enriquecedor de la propuesta a nivel creativo y compositivo.

- El modo en que las limitaciones de formato se convierten en posibilidades de reinterpretación y de libertad, reflejando bastante bien la dicotomía de los dispositivos de coerción y de creación que hemos explicitado en el tránsito que va de Foucault a Deleuze-Guattari.

- La necesidad de trabajar colaborativamente en torno a un mismo hecho que es la sesión en la caja de resonancia, en la que pueden intervenir programadores de código, ingenieros, artistas visuales, músicos o sonidistas, filósofos, psicólogos, etc. formando un equipo interdisciplinar que se tiene que escuchar mutuamente de manera constante; del mismo modo que si manejásemos una máquina o nave que nadie controla exclusiva 0 independientemente.

- La concentración que exige el espacio videovigilado al ser observado continuamente y por muchas perspectivas y niveles sin los ángulos muertos que deja por ejemplo un escenario convencional o incluso el cine o la televisión.

- $\quad$ Sin embargo, al mismo tiempo algunos participantes han destacado cierta intimidad que no se encuentra en un escenario; quizá por la complicidad que permite el trabajo en equipo.

Otro registro de resultados podemos encontrarlos en el impacto en redes sociales. Es una forma de medir los resultados relativa, pues no está claro hasta qué punto contrastan efectivamente un proyecto de este tipo. Deleuze-Guattari (2011) indican que justamente lo que se opone a la opinión es el arte, la ciencia y la filosofía; siendo su junción singular en el cerebro aquello que define la novedad frente a los objetos mentales pre-elaborados de la opinión. ¿Cuál es entonces el valor de las redes sociales? ¿Cómo nos relacionamos con los datos macro? ¿Cómo se difunde y se evalúa a este nivel el proyecto? Como se ha indicado anteriormente, los datos de redes sociales, tratados con herramientas de análisis de datos, pueden ser traídos a la propia caja de resonancia como se plantea en el espacio 5 de interacción siendo convertidos en fuentes o variables sensibles de tal modo que los pacientes pueden tocar e interactuar con ellos, teniendo más importancia como material de contra-efectuación que como información objetiva en sí misma, información que nos revele algún resultado.

Por tanto, la objetividad de los datos, aparte de su valor experiencial y expresivo, debe apuntar al modo en que podemos reorganizar las respuestas y cuadros clínicos de los pacientes sin conducirlos a las tipologías, sino al contrario, liberando sus rasgos y devenires de singularidad. Esto implicará diseño de cartografías (Guattari-Rolnik, 2006) en vez de cuadros cerrados en árboles clasificatorios; es decir, permitir espacios en los que trazar tendencias y estrategias, como decíamos, en vez de dar cajones predeterminados para llenar de casos.

\section{DISCUSIÓN}

Aunque se han planteado ya métodos artísticos como terapia en pacientes en psicología y medicina, es difícil encontrar un proyecto en el que se aúnen diferentes disciplinas (incluida la creación de hardware y programación de software) para un objetivo semejante. Normalmente la innovación creativa relacionada con artes visuales y los nuevos media, se plantean en un campo exclusivamente artístico, como un problema del arte contemporáneo por ejemplo, mientras que aquí se ve como parte de un dispositivo muy concreto. Este dispositivo no dejaría de ser sino la realización de un laboratorio de esquizoanálisis, tal como se puede entrever que plantean Deleuze-Guattari, aunque ampliando considerablemente su concepción y sus posibilidades al insertarlo en una pragmática efectiva que incluye no sólo el tratamiento digital del audiovisual y las conexiones en red, sino como hemos visto posibilidades de desarrollo mediante tratamiento de datos sociales y neuroimagen. Sin embargo, al mismo tiempo, vemos la necesidad de una síntesis semejante. El desarrollo de las tecnologías y su impacto social pone en cuestión nuestras relaciones grupales, personales e íntimas. De alguna manera, se pone en cuestión el propio cerebro, como receptor conectado a una gran red de mensajes que es Internet y todo lo que puede llevar asociado; es decir, el modo en que determina no sólo lo que pasa en Internet sino cada vez más el conjunto de nuestra vida y las cosas que utilizamos. En este contexto, ante este abismo, el cerebro, y también nuestro cuerpo, tiene que ser recuperado; tal como decía Artaud sobre el cerebro: "sus antenas vueltas hacia lo 
invisible"; su aptitud para "recomenzar una resurrección de la muerte" (Deleuze, 1984, p. 280). Sin necesidad de ser catastrofistas, necesitamos dispositivos que recuperen nuestras singularidades, la excepcionalidad de nuestros puntos de vista. No tenemos prácticamente dispositivos que operen en el sentido inverso del todo-individuo, ya que nuestros perfiles en redes sociales tienden más a diluir nuestros mensajes en los mares y océanos de la opinión que al contrario: caracterizar y singularizar nuestro punto de vista. Somos en este sentido más arrollados que supervivientes, quizá con la excepción de algunas celebridades que consiguen configurar a su alrededor un campo propio de enunciación. Pero, incluso en estos casos, el precio suele ser alto.

Al mismo tiempo no podemos negar la oportunidad que significan las TIC y los nuevos media para un proyecto semejante. La necesidad de aprender con todo el cerebro que desde campos intermediarios del arte y la psicología se viene manifestando hace ya décadas, es una consecuencia casi natural de las aplicaciones de estas nuevas TIC y los audiovisuales. Nuevo hardware, sensores, softwares de tracking video y reconocimiento, realidad aumentada, etc. más allá de su uso en dispositivos de control y vigilancia, tanto como dispositivos de mero entretenimiento y evasión, tienen que medir todavía su alcance clínico en la psicología personal y social. La relación del individuo con los dispositivos, estratos y archivos de su época, puede y debe conducir así a las ciencias humanas y sociales en su conjunto a definir sus nuevos laboratorios, en los que más allá de la documentación se alcance también experimentación que nos permita redefinirnos y reinventarnos continuamente, en vez de ser meros objetos o apéndices de nuestros desarrollos.

\section{AGRADECIMIENTOS}

Gracias al artista y programador Horacio González por ayudarme a desarrollar las tecnologías interactivas con OpenFrameworks y Arduino y a Irene del Valle, arquitecta, por la elaboración de los gráficos de este artículo.

\section{REFERENCIAS}

Baudrillard, J., \& Vazquez, J. (2001). Olvidar a Foucault. Valencia: Pre-Textos.

Bratton, B. H. (2015). The stack: on software and sovereignty. Cambridge, Massachusetts: MIT Press.
Deleuze, G. (1984). Estudios sobre cine. Barcelona: Paidós.

Deleuze, G. (1994). La imagen-movimiento: estudios sobre cine. Barcelona: Paidós.

Deleuze, G. (2011). Lógica del sentido. Barcelona: Paidós.

Deleuze, G., \& Guattari, F. (2002). Mil mesetas: capitalismo y esquizofrenia (5. ed). Valencia: Pre-Textos.

Deleuze, G., \& Guattari, F. (2005). Qu'est-ce que la philosophie? Paris: Editions de Minuit.

Deleuze, G., Guattari, F., \& Kauf, T. (2011). ¿Qué es la filosofía? Barcelona, España: Anagrama.

Deleuze, G., Guattari, F., \& Monge, F. (2017). El anti-edipo: capitalismo y esquizofrenia. Barcelona: Paidós.

Deleuze, G., Morey, M., \& Vazquez Pérez, J. (2003). Foucault.

Foucault, M. (2005). A arqueologia do saber. Coimbra: Almedina.

Gadamer, H. G. (2002). Verdad y metodo. España: Sigueme.

Guattari, F., \& Rolnik, S. (2006). Micropolítica: cartografías del deseo. Madrid: Traficantes de Sueños.

Lanier, J. (2011). You are not a gadget: a manifesto (publ. in Penguin books with updated material). London: Penguin Books.

Lévy, P. (1997). L'intelligence collective: pour une anthropologie du cyberspace. Paris: La Découverte.

Lévy, P. (1999). ¿Qué es lo virtual? Barcelona: Paidós.

Lyotard, J.-F. (1999). La condición postmoderna. Barcelona: Altaya.

Ortiz-Osés, A. (2003). Amor y sentido: una hermenéutica simbólica (1. ed). Rubí (Barcelona): Anthropos Editorial.

Ramey, J. A., \& Salzano, J. (2016). Deleuze hermético: filosofía y prueba espiritual. Buenos Aires: Editorial Las Cuarenta.

Schinca, M. (2011). Expresión corporal: técnica y expresión del movimiento. Las Rozas, Madrid: Wolters Kluwer Educación.

Schinca, M. (2011). Manual de psicomotricidad, ritmo y expresión corporal. Barcelona: Wolters Kluwer Educación.

Simondon, G., Martínez, M., \& Rodríguez, P. (2017). Sobre la técnica: (1953-1983). Buenos Aires: Cactus.

van den Heuvel, M. P., \& Sporns, O. (2013). Network hubs in the human brain. Trends in Cognitive Sciences, 17(12), 683-696. https://doi.org/10.1016/j.tics.2013.09.012

Vattimo, G. (1990). El fin de la modernidad: nihilismo y hermenéutica en la cultura posmoderna (3. ed). Barcelona: Gedisa.

Williams, L. V. (1986). Aprender con todo el cerebro. Barcelona: Ed. Martínez Roca. 